\title{
Bertolotti's Syndrome Requiring Intervention for Lower Back Pain: Two Cases Suspected as Ankylosing Spondylitis
}

\author{
Juyeon Kang, M.D. ${ }^{1}$, Seunghun Lee, M.D., Ph.D. ${ }^{2}$, Tae-Hwan Kim, M.D., Ph.D. ${ }^{3}$ \\ ${ }^{1}$ Department of Rheumatology, Inje University Busan Paik Hospital, Busan, ${ }^{2}$ Department of Radiology, Hanyang University College of Medicine, \\ Hanyang University Seoul Hospital, ${ }^{3}$ Department of Rheumatology, Hanyang University Hospital for Rheumatic Diseases, Seoul, Korea
}

\begin{abstract}
Bertolotti's syndrome is the part of lumbosacral transitional vertebra and is a congenital anomaly of the axial spine. The syndrome is associated with changes in the sacrum or iliac crest and secondary arthritis and is often mistaken for ankylosing spondylitis (AS). The mechanism of pain in this disease is unclear, and treatments have not been accurately established. Here, we report two cases of Bertolotti's syndrome in patients with severe back pain who were initially suspected to have AS and required interventional procedures. The two young adult patients developed symptoms similar to those of inflammatory back pain, and their symptoms did not improve with conventional treatment alone. Both patients underwent interventional management, and the symptoms improved. It may be clinically helpful to confirm the presence of Bertolotti's syndrome in patients with suspected AS, and interventional therapy may be required in some cases. (J Rheum Dis 2020;27:209-212)
\end{abstract}

Key Words. Bertolotti's syndrome, Ankylosing spondylitis, Lower back pain, Intervention

\section{INTRODUCTION}

Bertolotti's syndrome, first described in 1917 by an Italian physician called Mario Bertolotti [1] is a congenital abnormality in which the expanded lower lumbar transverse process articulates with ilium or sacrum $[2,3]$. The syndrome can be bilateral or unilateral and is an important cause of back pain, sometimes severe, especially in young patients. The prevalence of Bertolotti's syndrome is estimated to be about $4 \% \sim 30 \%$ in the general population, but the prevalence increases to $35.6 \%$ in patients who want treatment for lower back pain $[4,5]$. However, treatment guidelines for this disease have not yet been established, and affected patients receive symptomatic treatment in various fields of medicine, such as orthopedic surgery, pain clinic, and rheumatology.

Meanwhile, the main symptoms of ankylosing spondylitis (AS) also included lower back pain [6]. Patients with Bertolotti's syndrome and patients with AS both experi- ence disease onset at a young age [7], and the symptoms in both diseases of patients may be alleviated with non-steroidal anti-inflammatory drugs (NSAIDs). In particular, patients with AS may suffer decreased functional activity and reduced quality of life if proper treatment is not performed at the appropriate time, so accurate diagnosis and timely treatment are necessary [8-10]. We surveyed cases of Bertolotti's syndrome in patients who were initially suspected to have ankylosing spondylitis and were performed examinations. And we describe two patients with severe symptoms who improved with interventional management, intending to highlight the necessity of confirming Bertolotti's syndrome in patients with suspected ankylosing spondylitis.

\section{CASE REPORT}

\section{Case 1}

A 44-year-old female patient visited the rheumatology

\footnotetext{
Received : April 11, 2020, Revised : (1st) May 8, 2020,(2nd) May 10, 2020, Accepted : May 11, 2020

Corresponding to : Tae-Hwan Kim (1Dhttp://orcid.org/0000-0002-3542-2276

Department of Rheumatology, Hanyang University Hospital for Rheumatic Diseases, 222-1 Wangsimni-ro, Seongdong-gu, Seoul 04763, Korea. E-mail : thkim@hanyang.ac.kr
}

Copyright (c) 2020 by The Korean College of Rheumatology.

This is an Open Access article, which permits unrestricted non-commerical use, distribution, and reproduction in any medium, provided the original work is properly cited. 
department for back pain. The patient reported that symptoms first started four years before, and had recently worsened. The back pain was localized in the lower back and buttock, and frequently woke her from sleep. The pain intensity on visual analogue scale (VAS) was 7/10. In addition, she experienced an intermittent tingling symptom of the left leg. The pain was worse when changing posture. She had no familial history, no other comorbidities, and she was never smoker. The patient showed full muscle power of the bilateral lower extremities, and no sensory impairment was detected on physical examination. No abnormalities were found in laboratory tests.

Under the impression of AS, we performed further evaluations, the findings on X-rays were suspected Bertolotti's syndrome. It was identified as type II a, which was incomplete unilateral lumbarization/sacralization with an enlarged transverse process that has a diarthrosis between itself and the sacrum (Figure 1A), and which was finally confirmed through magnetic resonance imaging (MRI) (Figure 1B). Although the patient received medication for pain control, the symptoms persisted. So interventional treatment (fluoroscopic-guided steroid injection between the left enlarged transverse process of L5 vertebra and the ala of sacrum) was administered (Figure $1 C)$, and the pain was almost improved, but tingling sensation remained. The patient's overall symptoms improved with herniated lumbar disc treatment (physiotherapy, etc.) which had also been confirmed on MRI.

\section{Case 2}

An 18-year-old male patient visited to the rheumatology department for further evaluation of back pain. The symptom became severe one month previously, and he received treatment (sulfasalazine $500 \mathrm{mg}$ twice a day, meloxicam $7.5 \mathrm{mg}$ twice a day, and colchicine $0.6 \mathrm{mg}$ twice a day) for suspected AS or spondyloarthropathy at other hospital. He was referred to the tertiary hospital for persistent pain. The patient stated the pain was localized in the lower back and buttock and frequently woke him from sleep. The pain intensity on VAS was $8 / 10$. He had no familial history, no other comorbidities, and he had no smoking experience. On physical examination, the patient had normal muscle power of the bilateral lower extremities, and no sensory impairment was detected. There was no special provocation factor, and symptoms were getting worse despite changing medication (To add triamcinolone $2 \mathrm{mg}$ once a day, and change meloxicam to aceclofenac). There were no abnormalities were identified on the laboratory test.

Bertolotti's syndrome was suspected from X-rays (Figure 2A), also type II a, and cortical irregularity of the sacroiliac (SI) joint was also observed. In the delayed image of a bone scan, increased uptake was observed in the upper proportion of the SI joint (Figure 2B). In addition, single-photon emission computed tomography (SPECT) images demonstrated increased uptake in the left transitional vertebra (Figure $2 \mathrm{C}$ ). We diagnosed the cause of the patient's pain and performed an intervention procedure at pseudoarticulation site (fluoroscopic-guided steroid injection between enlarged left transverse process and sacrum) to improve the symptoms (Figure 2D).
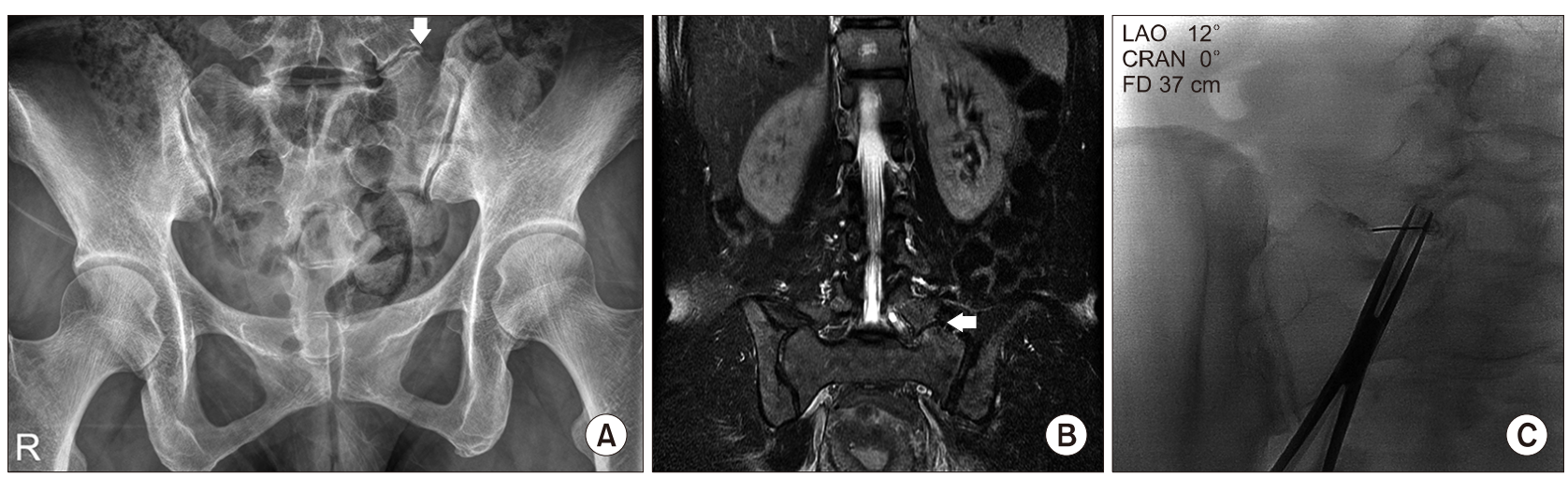

Figure 1. Diagnostic and interventional images of patient 1. Pelvis anteriorposterior (AP) radiograph showing a lumbosacral transition vertebra and the left upper border of sacrum are articulate (white arrow) (A), oblique coronal corresponding T2-weighted image of the sacral ala and the lower lumbar vertebra showing an inflammatory pseudoarthrosis (white arrow) between the left transverse process of $\mathrm{L} 5$ vertebra and the ala of sacrum (B) and fluoroscopic image of a contrast injection and steroid injection into an articulation between the left L5 to S1 transitional vertebra (C). 

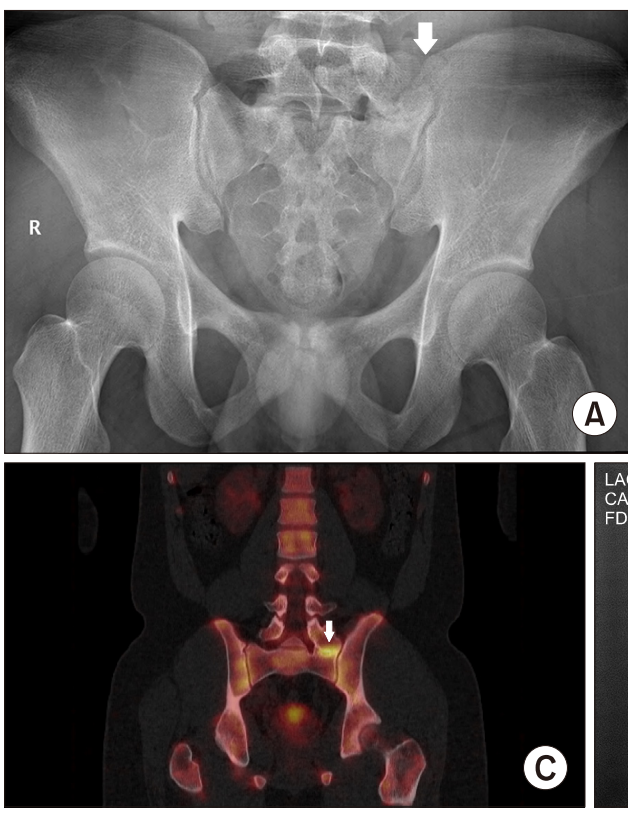

A

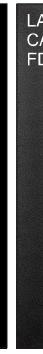

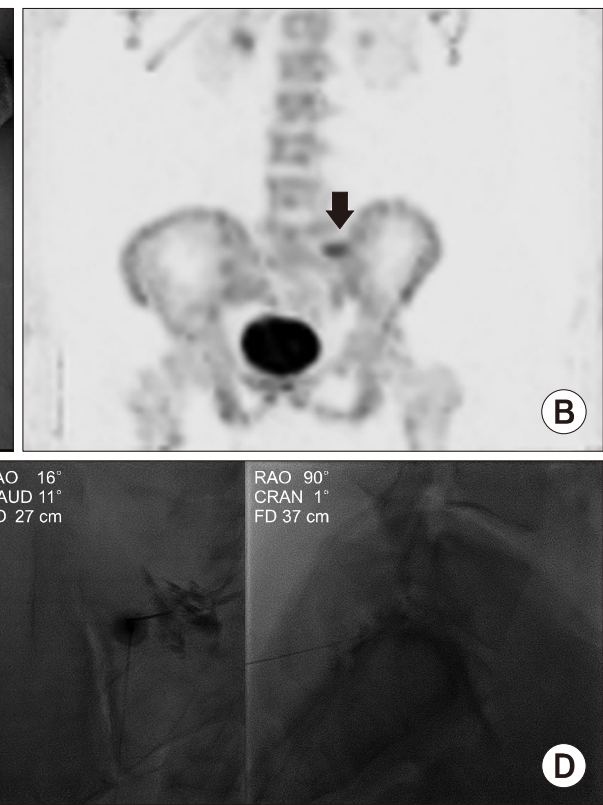

Figure 2. Diagnostic and interventional images of patient 2 . Pelvis anteriorposterior (AP) radiograph showing a left lumbosacral transition vertebra (white arrow) (A), an increased uptake (black arrow) is observed in the upper portion of the left sacroiliac joint in the delayed whole body bone scan image (B) and focal uptake in left transitional vertebra (white arrow) in single-photon emission computed tomography (SPECT) image (C). Fluoroscopic image of a contrast injection and steroid injection into between enlarged left transverse process and sacrum (D).

\section{DISCUSSION}

There are four types of lumbosacral transitional vertebrae, and various reports have described the histopathological and radiological changes that appear in the adjacent structures [11]. Type I exhibits unilateral (I a) or bilateral (I b) dysplastic transverse processes, measuring at least $19 \mathrm{~mm}$ at the craniocaudal axis. Type II includes incomplete unilateral (II a) or bilateral (II b) lumbarization/sacralization with an enlarged transverse process that has a diarthrosis between itself and the sacrum. Type III involves unilateral (III a) or bilateral (III b) lumbarization/sacralization with complete osseous fusion of the transverse process (es) to the sacrum. Type IV describes a unilateral Type II transition with the coexistence of Type III at the contralateral side. Among these, Types II, III, and IV are associated with Bertolotti's syndrome, and patient with Type I are usually asymptomatic [12].

The causes of pain in Bertolotti's syndrome are thought to vary but have not been clearly identified. Past studies suggested degenerative changes of the transverse process and ilium as a possible etiology of pain [13]. In addition, the incidence of degenerative disc disease and spinal stenosis was almost 9 times higher at the level adjacent to the transitional spine, suggesting that there may be more abnormal biomechanical stress than fusion $[3,11]$. Likewise, association has been reported between Bertolotti's syndrome and other diseases, such as intervertebral disc herniation and facet joint degeneration [14]. Another study suggested the cause of pain in Bertolotti's syn- drome as fused vertebrae causing instability compared to vertebrae with a normal structure [15]. Therefore, physicians can approach patients with Bertolotti's syndrome by considering its various etiologies and suggest the most appropriate treatment method, such as medication, interventional procedure, or surgery.

Both AS and Bertolotti's syndrome occur at a young age and the symptoms that patients initially complain are similar, so it is difficult to distinguish them from the symptoms alone. In addition, although these two diseases may coexist, there were few reports on how to distinguish which patient's pain is mainly due to which of the two. From March 2010 to March 2020, a total of 50 patients from our single tertiary institution was identified with Bertolotti's syndrome in the rheumatology division. Thirty-eight patients had back pain symptoms, and $17 \mathrm{pa}-$ tients were confirmed to have comorbid AS and Bertolotti's syndrome. Eleven of the remaining 21 Bertolotti's syndrome patients with back pain symptoms were initially suspected of having AS, but finally confirmed to have only Bertolotti's syndrome. Therefore, it would be of great clinical value to identify the presence of Bertolotti's syndrome as well as AS when attempting to determine the cause of lower back pain in a young patient. Bertolotti's syndrome can be confirmed by X-ray alone. However, to determine if the lesion is the actual cause of pain, it is useful to perform an MRI to find bone marrow edema caused by inflammation or to identify site of increased uptake due to inflammation through bone SPECT.

As the symptoms and severity of Bertolotti's syndrome 
vary, treatment methods are not clearly defined. Management of Bertolotti's syndrome should be initiated with a comprehensive conservative approach consisting of activity modification, physical therapy, and medications such as NSAIDs and a muscle relaxant. Interventional management can be beneficial in cases refractory to conservative management. For patients who present with lumbosacral radiculopathy or radiculitis symptoms due to direct compression or irritation of a nerve root by an enlarged transverse process or pseudoarticulation, a transforaminal or interlaminar epidural steroid injection maybe considered [5]. The patients in our cases did not respond to conventional treatment, and their pain improved after undergoing steroid injection. Therefore, when evaluating a patient with severe symptoms caused by Bertolotti's syndrome, it may be a good choice to consider interventional treatment a priority. However, if the patient's symptoms do not respond to these non-surgical treatments, surgical resection is needed [13].

Future studies in a larger number of patients are necessary to identify the characteristics of Bertolotti's syndrome with or without AS, as are studies that approach molecular and genetic levels for development of various treatments.

\section{SUMMARY}

When evaluating a patient with suspected AS in the outpatient setting, confirming the presence of Bertolotti's syndrome will be clinically helpful. In addition, if the symptoms cannot be alleviated by conventional treatment, interventional management should be considered.

\section{CONFLICT OF INTEREST}

No potential conflict of interest relevant to this article was reported.

\section{AUTHOR CONTRIBUTIONS}

J.K. and T.-H.K. created the concept and design of the study, and S.L. performed interpretation of the imaging data. J.K. wrote the manuscript and prepared the figures. T.-H.K. performed critical revision of the manuscript. All authors read and approved the final manuscript.

\section{REFERENCES}

1. Bertolotti M. Contributo alla conoscenza dei vizi di differenzazione regionale del rachide con speciale riguardo all assimilazione sacrale della v. lombare. Radiol Med 1917;4: 113-44.

2. Apazidis A, Ricart PA, Diefenbach CM, Spivak JM. The prevalence of transitional vertebrae in the lumbar spine. Spine J 2011;11:858-62.

3. Quinlan JF, Duke D, Eustace S. Bertolotti's syndrome. A cause of back pain in young people. J Bone Joint Surg Br 2006;88:1183-6.

4. Delport EG, Cucuzzella TR, Kim N, Marley J, Pruitt C, Delport AG. Lumbosacral transitional vertebrae: incidence in a consecutive patient series. Pain Physician 2006;9:53-6.

5. Iannuccilli JD, Prince EA, Soares GM. Interventional spine procedures for management of chronic low back pain-a primer. Semin Intervent Radiol 2013;30:307-17.

6. Braun J, Sieper J. Ankylosing spondylitis. Lancet 2007;369: 1379-90.

7. Jancuska JM, Spivak JM, Bendo JA. A review of symptomatic lumbosacral transitional vertebrae: Bertolotti's syndrome. Int J Spine Surg 2015;9:42.

8. Bostan EE, Borman P, Bodur H, Barça N. Functional disability and quality of life in patients with ankylosing spondylitis. Rheumatol Int 2003;23:121-6.

9. Chorus AM, Miedema HS, Boonen A, Van Der Linden S. Quality of life and work in patients with rheumatoid arthritis and ankylosing spondylitis of working age. Ann Rheum Dis 2003;62:1178-84.

10. Cakar E, Taskaynatan MA, Dincer U, Kiralp MZ, Durmus O, Ozgül A. Work disability in ankylosing spondylitis: differences among working and work-disabled patients. Clin Rheumatol 2009;28:1309-14.

11. Castellvi AE, Goldstein LA, Chan DP. Lumbosacral transitional vertebrae and their relationship with lumbar extradural defects. Spine (Phila Pa 1976) 1984;9:493-5.

12. Konin GP, Walz DM. Lumbosacral transitional vertebrae: classification, imaging findings, and clinical relevance. AJNR Am J Neuroradiol 2010;31:1778-86.

13. Manmohan S, Dzulkarnain A, Nor Azlin ZA, Fazir M. Bertolotti's syndrome: a commonly missed cause of back pain in young patients. Malays Fam Physician 2015;10:55-8.

14. Vergauwen S, Parizel PM, van Breusegem L, Van Goethem JW, Nackaerts Y, Van den Hauwe L, et al. Distribution and incidence of degenerative spine changes in patients with a lumbo-sacral transitional vertebra. Eur Spine J 1997;6: 168-72.

15. Chiang GT, Anuar MS, Ibrahim K, Baharudin AB, Ariffin $\mathrm{MH}$. A case reports of intra-articular steroid injection in the treatment of Bertolotti's syndrome. Asian J Orthop Res 2018;1:1-6. 